\title{
Melanisation of Aspergillus terreus-Is Butyrolactone I Involved in the Regulation of Both DOPA and DHN Types of Pigments in Submerged Culture?
}

\author{
Elina K. Palonen ${ }^{1, *}$, Sheetal Raina ${ }^{2}$, Annika Brandt ${ }^{1}$, Jussi Meriluoto ${ }^{1}$, Tajalli Keshavarz ${ }^{2}$ \\ and Juhani T. Soini ${ }^{3}$ \\ 1 Biochemistry, Faculty of Science and Engineering, Åbo Akademi University, Artillerigatan 6, \\ Åbo FI-20520, Finland; annika.brandt@abo.fi (A.B.); jussi.meriluoto@abo.fi (J.M.) \\ 2 Department of Life Sciences, University of Westminster, London W1W 6UW, UK; \\ rainas2@googlemail.com (S.R.); t.keshavarz@westminster.ac.uk (T.K.) \\ 3 Faculty of Life Sciences and Business, Turku University of Applied Sciences, Lemminkäinengatan 30, \\ Åbo FI-20520, Finland; juhani.soini@turkuamk.fi \\ * Correspondence: elina.palonen@abo.fi; Tel.: +358-4-4270-2324
}

Academic Editors: Annele Hatakka and Taina Lundell

Received: 17 March 2017; Accepted: 28 April 2017; Published: 4 May 2017

\begin{abstract}
Pigments and melanins of fungal spores have been investigated for decades, revealing important roles in the survival of the fungus in hostile environments. The key genes and the encoded enzymes for pigment and melanin biosynthesis have recently been found in Ascomycota, including Aspergillus spp. In Aspergillus terreus, the pigmentation has remained mysterious with only one class of melanin biogenesis being found. In this study, we examined an intriguing, partially annotated gene cluster of $A$. terreus strain NIH2624, utilizing previously sequenced transcriptome and improved gene expression data of strain MUCL 38669, under the influence of a suggested quorum sensing inducing metabolite, butyrolactone I. The core polyketide synthase (PKS) gene of the cluster was predicted to be significantly longer on the basis of the obtained transcriptional data, and the surrounding cluster was positively regulated by butyrolactone I at the late growth phase of submerged culture, presumably during sporulation. Phylogenetic analysis of the extended PKS revealed remarkable similarity with a group of known pigments of Fusarium spp., indicating a similar function for this PKS. We present a hypothesis of this PKS cluster to biosynthesise a 1,8-dihydroxynaphthalene (DHN)-type of pigment during sporulation with the influence of butyrolactone I under submerged culture.
\end{abstract}

Keywords: Aspergillus terreus; filamentous fungi; pigment; melanin; butyrolactone I; transcriptome sequencing; gene expression; NR-PKS cluster

\section{Introduction}

The filamentous fungi of Aspergillus species are known to cause several diseases and to contain pathogenic features with objects ranging from plants to humans. These fungal features include resistance to environmental damage sources such as UV, heat, detergents, phagocytosis and antimicrobial drugs. One of the factors involved in these antagonistic properties is fungal sporulation and the corresponding resistance improving characteristics of the spores, including pigments and melanins [1-6]. The biosynthesis pathways of fungal pigment and melanin polymers have been divided into two classes, DHN (1,8-dihydroxynaphthalene) or DOPA (3,4-dihydroxyphenylalanine) pathways. The classification of these types is, however, diverged into two methods, either based on the identification of the pathway intermediates, or on the observed effects of applying specific 
intermediate enzyme inhibitors. Specifically, the DHN classification is either based on the identification of naphthopyrone precursors or on the effect of inhibitors-tricyclazole or phthalide-targeted to hydroxynaphthalene reductase with classical short-chain dehydrogenase/reductase (SDR) and Rossmann fold domains. The biogenesis of this type of melanin usually begins with polyketide synthesis, followed by tailoring steps and polymerisation. The classification of the other melanin type, DOPA, is based on the presence of tyrosine or 3,4-dihydroxyphenylalanine precursors, or on the involvement of tyrosinase enzyme, indicated with inhibitors kojic acid and tropolone. The resulting melanin intermediates of this DOPA pathway are commonly polymerised as well [6-10]. So far, the biosynthesis and polymerisation pathways as well as the specific morphological locations of diverse pigments are still unrevealed concerning many filamentous fungi. However, both DHN-type of conidial pigments and DOPA-type of melanins have been discovered in few Aspergillus species, although the DHN-type of pigments are presumed to be more common [6,11-14].

The key enzymes in producing pigments and melanins in Aspergillus terreus have been searched for in several studies with no resulting identifications regarding polyketide synthases (PKS) [14-16], as far as we know. In contrast, a non-ribosomal peptide synthetase (NRPS)-like enzyme MelA was revealed to synthesise an uncommon aspulvinone E-derived Asp-melanin together with a tyrosinase TyrP $[17,18]$, indicating the production to be of DOPA-type. Nevertheless, the pigmentation pathways of A. terreus are still mysterious, being only partially discovered. Pal et al. demonstrated the presence of a DHN-type of pigment using specific inhibitors, both on static culture as well as in submerged growth conditions [14]. Intriguingly, Schimmel et al. reported increased sporulation along with secondary metabolism of $A$. terreus in submerged culture conditions, as a result of supplementing butyrolactone I, which was later implicated as a quorum sensing inducing molecule in A. terreus [19-21]. Butyrolactone I was recently suggested to be involved in the gene expression control of the key regulators of conidiation ( $b r l A, a b a A$ and $\operatorname{wet} A)$ as well as the global regulator laeA [22] in accordance with the previous study of Schimmel et al. [19]. However, pigmentation has not been examined in the same growth conditions, to our knowledge.

In this study, we describe a potential, non-reducing polyketide synthase (NR-PKS) with a non-canonical domain structure within Aspergilli, and the surrounding gene cluster, which were revealed through an in-depth analysis of the recently sequenced transcriptome of $A$. terreus strain MUCL 38669 [22]. The gene cluster transcripts appeared to be only partially annotated in the A. terreus strain NIH2624, leading to a further examination of the transcripts. We present a hypothesis of the function for this gene cluster to be involved in a DHN-type pigmentation pathway, in advance of the necessary further investigations. We display the transcriptional levels and gene expression profiles of both the known DOPA-like as well as the suggested DHN-like pigment clusters during the developmental growth phases of the submerged A. terreus culture. We suggest that butyrolactone I, as an indicated quorum sensing molecule, plays a regulative role in the pigmentation processes based on large-scale gene expression and whole transcriptome sequencing results.

\section{Materials and Methods}

\subsection{Strain, Chemicals and Culture Conditions}

All culture materials, including the Aspergillus terreus strain MUCL 38669, are the same as was used previously [20-22]. A. terreus MUCL 38669 was cultured under shaken, submerged growth conditions in three biological replicates for nine days. The growth conditions were the same as in previous studies of secondary metabolism [20,21]. Shortly, A. terreus MUCL 38669 spores were maintained on yeast and malt extract (YME) agar slants. Collected spores (final concentration $10^{7} / \mathrm{mL}$ ) had been incubated in $100 \mathrm{~mL}$ of inoculation medium for $25 \mathrm{~h}$ at $27^{\circ} \mathrm{C}$. In addition, $100 \mathrm{~mL}$ of glucose, peptonised milk, yeast extract and lactose containing (GPY-L) production medium ( $\mathrm{pH} 7.4$ ) was inoculated with $10 \mathrm{~mL}$ of the inoculation medium and incubated at $27^{\circ} \mathrm{C}$ for $216 \mathrm{~h}$. 
Addition of Butyrolactone I

Exogenous butyrolactone I was added at $24 \mathrm{~h}, 96 \mathrm{~h}$ and $120 \mathrm{~h}$ post inoculation (test sets 1, 2 and 3, respectively) and each of the test sets as well as the control set (no butyrolactone I added) were sampled at $24 \mathrm{~h}, 48 \mathrm{~h}, 96 \mathrm{~h}, 120 \mathrm{~h}, 144 \mathrm{~h}$ and $216 \mathrm{~h}$ post inoculation. The exogenous butyrolactone I was dissolved in ethanol and added to the test cultures to a final concentration of $100 \mathrm{nM}$.

\subsection{Gene Expression Analysis Using Microarrays}

The total RNA was derived from a previous study [20] where A. terreus mycelia was sampled at the 6 time points and was stored at $-80^{\circ} \mathrm{C}$. The microarray gene expression data was obtained and analysed as described in our related study [22]. The analysis contained one unusual step regarding the 60-mer oligonucleotide microarray probes that had been designed based on the available genomic sequence of strain NIH2624 [23]. Additional bioinformatic steps were applied to exclude the unreliable probes by alignment based filtering using BLASTN software (version 2.2.29+, National Centre for Biotechnology Information (NCBI), Bethesda, MD, USA) [24,25] prior to the further deep analysis, due to the observed nucleotide-level differences between the A. terreus strains NIH2624 and MUCL 38669 (details in our related study [22]). The microarray experiment was performed in three biological replicates and in four technical replicates on probe level. Differential gene expression $\left(\left|\log _{2} \mathrm{FC}\right|>0.5\right)$ was considered as statistically significant if adjusted $p<0.05$.

\subsection{Strand-Specific Transcriptome Sequencing and Analysis}

The assembled transcriptome sequence data was obtained from our related study [22]. Shortly, the method used for the synthesis of strand-specific double stranded cDNA (ds cDNA) from the total RNA was a modified combination of protocols presented by Marioni et al. [26], Parkhomchuk et al. [27], Levin et al. [28] and the standard Illumina mRNA preparation protocol (Preparing Samples for Sequencing of mRNA, Part No. 1004898 Rev. A, Illumina Inc., San Diego, CA, USA) due to the lack of strand-specificity in the contemporary standard mRNA sequencing protocols. The total RNA to be sequenced was extracted from the $A$. terreus mycelia samples taken at six time points of the culture where butyrolactone I had been added at $120 \mathrm{~h}$ p.i., obtained from the submerged culture of a previous study [20]. This set of total RNA was pooled to acquire the required amount of strand-specific ds cDNA for the high throughput DNA sequencing. The detailed description of the RNA preparation and the sequence assembly is explained in our related study [22].

The transcriptome analysis was performed as in our related study [22] and composed of the following steps, in short. The obtained transcripts along with the obtained sequence reads of A. terreus MUCL 38669 were aligned with the strain NIH2624 annotated genome and viewed using Integrative Genomics Viewer (IGV) (version IGV_2.3.23, Broad Institute, Cambridge, MA, USA) [29-31]. The resulting mapped reads were quantified and normalised using Cufflinks tools Cuffquant and Cuffnorm (version 2.2.1, University of Washington, Seattle, WA, USA) [32], giving the number of fragments per kilobase of exon per million reads mapped (FPKM), representing the accumulation of the transcripts of the pooled RNA samples (pooled FPKM).

\subsection{Data Availability}

The resulting complete transcript sequences analysed in this study were deposited at the National Centre for Biotechnology Information (NCBI) GeneBank database under the following accession numbers: KX470747 (pgmB), KX470748 (pgmD), KX470749 (pgmE), KX470750 (pgmF), KX470751 (pgmG), KX470752 (pgmH), and KX470753 (melA). The putative PKS sequence, pgmA, is deposited as a third-party annotation with the accession number BK009975. The raw transcriptome sequence data and microarray gene expression raw data of A. terreus MUCL 38669 have been submitted to the Sequence Read Archive (SRA) and Gene Expression Omnibus (GEO) databases of NCBI. The obtained accession numbers are PRJNA360953 (BioProject) and GSE93552 (GEO database). 


\section{Results}

\subsection{A Non-Canonical PKS Gene Cluster in A. terreus}

Exogenous supplementation of butyrolactone I has been suggested to increase sporulation under submerged growth conditions [19] and was recently further investigated on a transcriptomic level in our related study [22]. While analysing the sequenced transcriptome in order to illuminate the predicted secondary metabolite clusters, an intriguing cluster was encountered, containing an only partially annotated core PKS gene (ATEG_06206 in strain NIH2624, subsequently called pgmA). The transcriptome sequence data indicated a significantly longer transcript for this PKS gene. A computational GENSCAN [33] analysis of the surrounding genomic region of the PKS gene revealed the length of this open reading frame (ORF) to be $7232 \mathrm{bp}$ in accordance with the indicated length of the partially sequenced transcript (Figure 1 and Table 1). Translation using ExPASy Web Server [34] and the subsequent InterPRO protein domain prediction [35] revealed the protein to contain several domains that classify this PKS protein as a non-reducing polyketide synthase. The domain structure prediction revealed following domains: starter unit:ACP transacylase domain (SAT) on N-terminus, beta-ketoacyl synthase domain (KS), acyl transferase domain (AT), polyketide product template domain (PT), two successive acyl carrier domains (ACP) and thioester reductase domain (R) in the C-terminus, suggesting an exquisite NR-PKS subclass amongst Aspergilli (Figure 1).

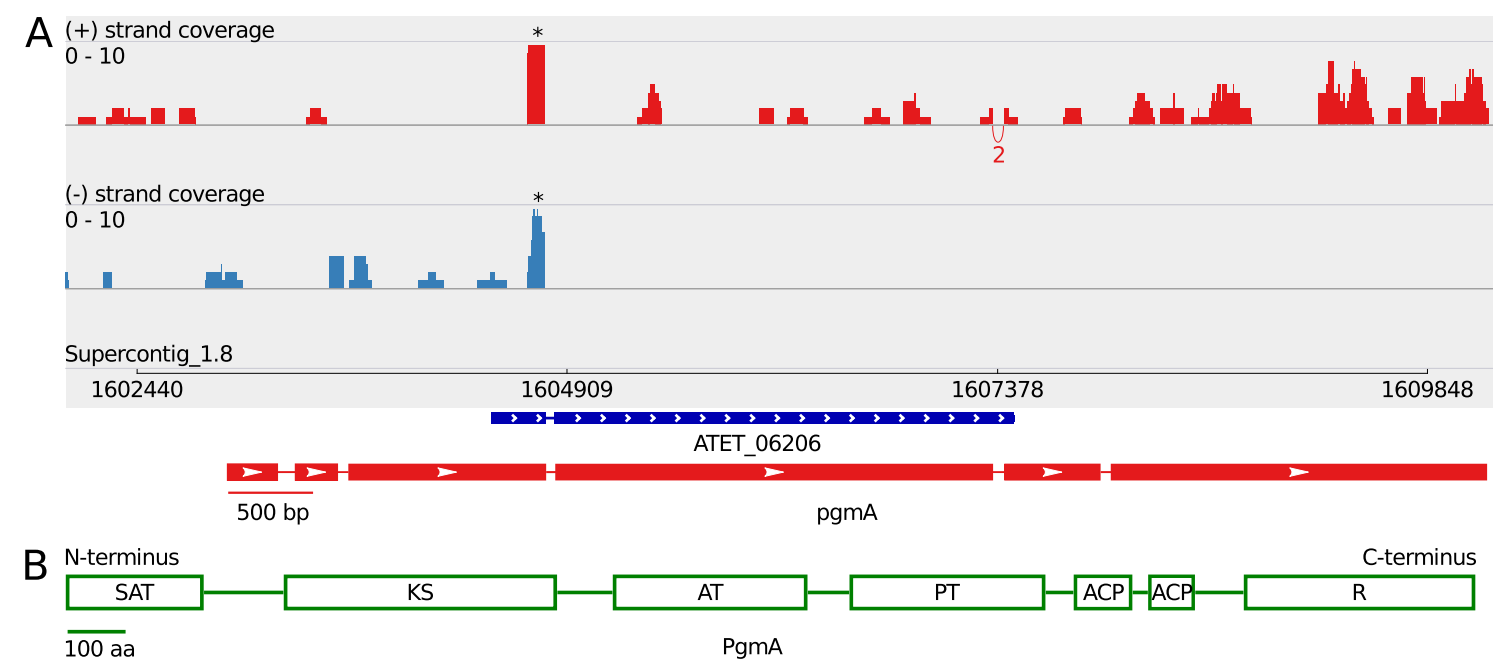

Figure 1. The predicted non-reducing polyketide synthase PgmA. (A) sashimi plot of the pgmA gene describing the strand-specific alignment coverage of the read sequences of A. terreus MUCL 38669 over the corresponding genomic region of NIH2624 supercontig_1.8, which was obtained using the Integrative Genomics Viewer (IGV) software [29-31]. The curved splice junctions represent the number of spliced reads indicating an intron at that specific location. The predicted gene structure is shown below the sashimi plot in the same scale as the plot; (B) the predicted protein domains of the translated sequence of PgmA: Starter unit:ACP transacylase (SAT), Beta-ketoacyl synthase (KS), Acyl transferase (AT), Polyketide product template (PT), Acyl carrier (ACP), Thioester reductase (R). * These coverage peaks are due to an erroneously aligned short part of a longer transcript located to a gene ATEG_06102.

A BLASTP search [36] displayed good similarity with a group of NR-PKS proteins of Fusarium species as the best match along with Aspergillus lentulus and Aspergillus glaucus, having residue identities between $58 \%$ and $63 \%$ and full-length alignment. A closer look at these BLASTP results revealed all of the proteins in this group to have the same domain structure with the PgmA protein whereas only A. lentulus and A. glaucus amongst Aspergillus species has a PKS protein with a similar domain structure to our knowledge. Four of the proteins of the group of Fusarium NR-PKS proteins with the same domain structure are known to produce perithecium 
pigments [37,38]. Specifically, these proteins are F. graminearum Pgl1 (XP_011328597.1), F. fujikuroi fusarubin producing Fsr1 (CCT64762.1), F. verticillioides Pgl1 (EWG41617.1) and F. solani Pgl1 (XP_003039929.1). A phylogenetic study of KS domains of several Ascomycete NR-PKS proteins, PgmA included, revealed homology to these core PKS enzymes of perithecium pigment biosynthesis (Figure 2), predicting a similar function to A. terreus PgmA protein. A closer look on the surrounding genes of $p g m A$ revealed a cluster of nine genes in total with predicted domains that are common for enzymes in secondary metabolites producing clusters (Tables 1 and 2). Each of the cluster genes has a complete or partial transcript coverage, indicating co-expression of these genes and some activity for this cluster in these growth conditions while the genes up- and downstream of this cluster displayed no coverage, confirming the cluster form (Figures S1-S3).

Table 1. Information on the A. terreus pgm gene cluster and the DOPA type melanin genes.

\begin{tabular}{ccccc}
\hline Gene & $\begin{array}{c}\text { Gene }^{\text {a }} \\
\text { Length (bp) }\end{array}$ & $\begin{array}{c}\text { ORF } \\
\text { Length (bp) }\end{array}$ & $\begin{array}{c}\text { Pooled } \\
\text { FPKM }\end{array}$ & $\begin{array}{c}\text { Coverage Max. } \\
\text { Sense Strand }\end{array}$ \\
\hline pgmB & $1448^{\mathrm{d}}$ & 1329 & 3.9 & $23^{\mathrm{c}}$ \\
pgmC & $1443^{\mathrm{d}}$ & $1443^{\mathrm{d}}$ & 1.1 & 6 \\
pgmR $^{\mathrm{e} m}$ & $1284^{\mathrm{e}}$ & $1284^{\mathrm{e}}$ & 0.13 & 2 \\
pgmA & $7232^{\mathrm{e}}$ & $6897^{\mathrm{e}}$ & 0.65 & 8 \\
pgmD & 1003 & 939 & 4.7 & $44^{\mathrm{c}}$ \\
pgmE & 1008 & 1008 & 15 & $91^{\mathrm{c}}$ \\
pgmF & 1071 & 1071 & 290 & $1634^{\mathrm{c}}$ \\
pgmG & 1967 & 1683 & 56 & $333^{\mathrm{c}}$ \\
pgmH & 1714 & 1479 & 12 & $81^{\mathrm{c}}$ \\
$m e l A$ & 2779 & 2779 & 6.0 & $38^{\mathrm{c}}$ \\
tyrP & $1230^{\mathrm{d}}$ & $1071^{\mathrm{d}}$ & 1.3 & $14^{\mathrm{C}}$ \\
\hline
\end{tabular}

${ }^{a}$ based on the sequenced ORF's $5^{\prime}$ and $3^{\prime}$ locations on the A. terreus NIH2624 genome assembly; ${ }^{b}$ represents the number of overlapping reads' nucleotides at one nucleotide site of the transcript obtained from the pooled RNA samples; ${ }^{c}$ complete read coverage; ${ }^{d}$ confirmed with GENSCAN Web Server [33]; ${ }^{\text {e }}$ predicted with GENSCAN Web Server [33]; DOPA: 3,4-dihydroxyphenylalanine; ORF: open reading frame; FPKM: fragments per kilobase of exon per million reads mapped.

A further BLASTP analysis also revealed similarity with the fusarubin biosynthesis cluster members of F. fujikuroi, having some genes in common with this cluster of $A$. terreus, in addition to the predicted core biosynthesis PKS gene. Specifically, the clusters have a putative O-methyltransferase ATEG_06203 (subsequently called pgmB), a putative quinone reductase ATEG_06209 (subsequently called $p g m F$ ) and a transcription factor ATEG_06205 (subsequently called pgmR) with similarity on the amino acid level and additionally a putative short-chain dehydrogenase/reductase ATEG_06207 (subsequently called $\mathrm{pg} m \mathrm{D}$ ) with a shared domain structure (Table 2). The central, multi-functional tailoring enzyme of the fusarubin cluster, Fsr3 with a flavin adenine dinucleotide (FAD) binding monooxygenase domain, has no clearly orthologous enzyme in this $A$. terreus cluster, whereas the cluster includes a putative cytochrome p450 monooxygenase encoding gene ATEG_06204 (subsequently called $\mathrm{pgm} C$ ), possibly having a similar function in the A. terreus biosynthesis pathway (Table 2). The A. terreus cluster appears to include three additional genes when compared to fusarubin biosynthesis gene cluster, namely, a putative S-adenosyl-L-methionine (SAM)-dependent methyltransferase ATEG_06208 (subsequently called pgmE), a putative major facilitator superfamily (MFS) permease ATEG_06210 (subsequently called pgmG) and a putative FAD-binding CO dehydrogenase ATEG_06211 (subsequently called pgmH) (Table 2). Taken together, we report a transcriptionally updated and computationally confirmed gene cluster annotation containing a non-canonical NR-PKS pgmA, being similar to pigment biosynthases amongst Fusarium species on protein level. All of these cluster genes being co-expressed suggests a secondary metabolite, possibly a pigment, to be biosynthesised in these submerged growth conditions under enhanced butyrolactone I influence. 


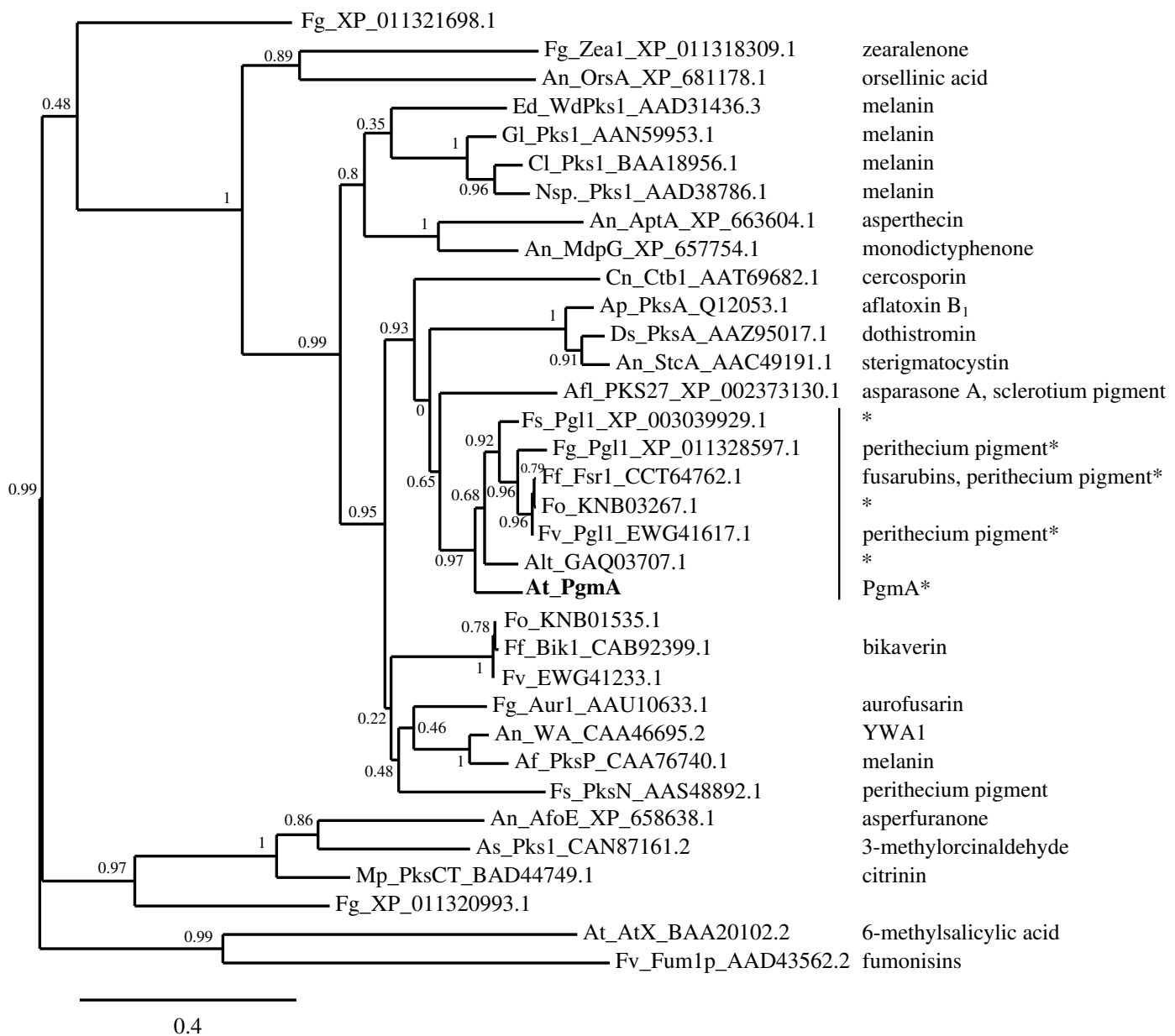

Figure 2. Phylogeny of different fungal polyketide synthases including pigment synthases. The range of polyketide synthases chosen for this analysis is based on a study of Studt et al. [37]. The extracted ketoacyl synthase domains of the polyketide synthases were compared and aligned to obtain a phylogram as described in our related study [22]. The chemical product of the corresponding polyketide synthase (PKS) is given on the right side if characterised, to our knowledge. The scale bar represents a 0.4 residue change per site. No outgroup was used. The organisms used in the phylogram: Af: A. fumigatus, Afl: A. flavus, Alt: A. lentulus, An: A. nidulans, Ap: A. parasiticus, As: Acremonium strictum, At: A. terreus, $\mathrm{Cl}$ : Colletotrichum lagenaria, Cn: Cercospora nicotianae, Ds: Dothistroma septosporum, Ed: Exophiala dermatitidis, Ff: Fusarium fujikuroi, Fg: F. graminearum, Fo: F. oxysporum, Fs: F. solani, Fv: F. verticillioides, Gl: Glarea lozoyensis, Mp: Monascus purpureus, Nsp.: Nodulisporium sp. strain ATCC74245. The protein sequences (except for the PgmA) were obtained from the following databases: National Centre for Biotechnology Information (NCBI) RefSeq, GenBank, DNA Data Bank of Japan (DDBJ), European Molecular Biology Laboratory (EMBL) and UniProtKB. * PKS domain structure is: SAT-KS-AT-PT-ACP-ACP-R. 
Table 2. Amino acid identity and InterPRO domains of the A. terreus Pgm cluster and F. fujikuroi fusarubin cluster ${ }^{\mathrm{a}}$.

\begin{tabular}{|c|c|c|c|c|c|}
\hline Gene & $\begin{array}{l}\text { Predicted Molecular } \\
\text { Function }\end{array}$ & $\begin{array}{l}\text { InterPRO } \\
\text { Annotation }\end{array}$ & Domains & ID \% & $\begin{array}{c}\text { Fusarubin } \\
\text { Gene }\end{array}$ \\
\hline PgmB & O-methyltransferase & $\begin{array}{l}\text { IPR016461 }^{\mathrm{d}} \\
\text { IPR011991 }^{\mathrm{d}} \\
\text { IPR029063 }^{\mathrm{d}} \\
\text { IPR001077 }^{\mathrm{d}}\end{array}$ & $\begin{array}{l}\text { O-methyltransferase, } \\
\text { COMT-type }\end{array}$ & 41 & Fsr $2^{f}$ \\
\hline $\operatorname{PgmC}^{b}$ & $\begin{array}{l}\text { Cytochrome p450 } \\
\text { monooxygenase }\end{array}$ & $\begin{array}{l}\text { IPR001128 } \\
\text { IPR002401 } \\
\text { IPR017972 }\end{array}$ & $\begin{array}{l}\text { Cytochrome P450 } \\
\text { E-class, group } 1\end{array}$ & $\mathrm{NA}^{\mathrm{e}}$ & Fsr3 $g$ \\
\hline $\operatorname{Pgm} R^{b}$ & $\begin{array}{l}\text { Aflatoxin biosynthesis } \\
\text { regulatory protein-like }\end{array}$ & $\begin{array}{l}\text { IPR002409 } \\
\text { IPR001138 }\end{array}$ & $\begin{array}{l}\text { Aflatoxin biosynthesis } \\
\text { regulatory protein }\end{array}$ & 31 & Fsr6 ${ }^{\mathrm{h}}$ \\
\hline $\operatorname{PgmA} b, c$ & $\begin{array}{l}\text { nonreducing polyketide } \\
\text { synthase; NR-PKS }\end{array}$ & $\begin{array}{l}\text { IPR032088 }^{\mathrm{d}} \\
\text { IPR020841 }^{\mathrm{d}} \\
\text { IPR020801 }^{\mathrm{d}} \\
\text { IPR030918 }^{\mathrm{d}} \\
\text { IPR009081 }^{\mathrm{d}} \\
\text { IPR01312 }^{\mathrm{d}}\end{array}$ & $\begin{array}{c}\text { Starter unit:ACP } \\
\text { transacylase } \\
\text { Beta-ketoacyl synthase } \\
\text { Acyl transferase } \\
\text { Polyketide product template } \\
\text { Acyl carrier protein-like } \\
\text { Thioester reductase-like }\end{array}$ & 59 & Fsr1 ${ }^{i}$ \\
\hline PgmD & $\begin{array}{c}\text { short-chain } \\
\text { dehydrogenase/reductase }\end{array}$ & $\begin{array}{l}\text { IPR002347 } \\
\text { IPR016040 }\end{array}$ & $\begin{array}{c}\text { Short-chain } \\
\text { dehydrogenase/reductase }\end{array}$ & $\mathrm{NA}^{\mathrm{e}}$ & Fsr $5^{\mathrm{j}}$ \\
\hline PgmE & $\begin{array}{l}\text { SAM-dependent } \\
\text { methyltransferase }\end{array}$ & IPR029063 & $\begin{array}{l}\text { SAM-dependent } \\
\text { methyltransferase }\end{array}$ & NA & NA \\
\hline PgmF & quinone reductase & $\begin{array}{l}\text { IPR002085 } 5^{\mathrm{d}} \\
\text { IPR011032 }^{\mathrm{d}} \\
\text { IPR020843 } \\
\text { IPR013154 } \\
\text { IPR }^{\mathrm{d}} \\
\end{array}$ & $\begin{array}{l}\text { Alcohol dehydrogenase } \\
\text { GroES-like } \\
\text { Enoylreductase domain } \\
\text { Alcohol dehydrogenase }\end{array}$ & 26 & Fsr4 ${ }^{\mathrm{k}}$ \\
\hline PgmG & MFS family permease & $\begin{array}{l}\text { IPR011701 } \\
\text { IPR020846 }\end{array}$ & Major facilitator superfamily & $\mathrm{NA}^{\mathrm{e}}$ & $\mathrm{NA}^{\mathrm{e}}$ \\
\hline PgmH & $\begin{array}{l}\text { FAD/FMN-binding } \\
\text { CO dehydrogenase }\end{array}$ & $\begin{array}{l}\text { IPR016167 } \\
\text { IPR016166 } \\
\text { IPR006094 } \\
\text { IPR016169 }\end{array}$ & $\begin{array}{c}\text { FAD-binding, type } 2 \\
\text { FAD linked oxidase } \\
\text { CO dehydrogenase flavoprotein- } \\
\text { like, FAD-binding, subdomain } 2\end{array}$ & $\mathrm{NA}^{\mathrm{e}}$ & $\mathrm{NA}^{\mathrm{e}}$ \\
\hline
\end{tabular}

\footnotetext{
${ }^{a}$ pgm cluster genes were translated using ExPASy, the domains were predicted using InterPRO and the amino acid identities were obtained using BLASTP Web Servers [34-36,39,40]; ${ }^{\mathrm{b}}$ the nucleotide sequence was obtained from GenBank database (strain NIH2624) and modified with GENSCAN [33]; ${ }^{c}$ Additional InterPRO domains of PgmA: IPR014030, IPR016039, IPR014031, IPR016035, IPR001227, IPR014043, IPR016036, IPR020807, IPR016040, IPR018201; ${ }^{\mathrm{d}}$ shared predicted domain; ${ }^{\mathrm{e}}$ not in the vicinity of the cluster or core biosynthesis gene; ${ }^{\mathrm{f}}$ Accession: CCT64761.1; ${ }^{\mathrm{h}}$ Accession: CCT64758.1; ${ }^{\mathrm{i}}$ Accession: CCT64762.1; ${ }^{\mathrm{j}}$ Accession: CCT65046.1; ${ }^{\mathrm{k}}$ Accession: CCT64759.1; ${ }^{\mathrm{g}}$ Accession: CCT64760.1, PgmC and Fsr3 share the same GeneOntology prediction: oxidation reduction process (GO:0055114) and a monooxygenase motif; NA, no significant BLASTP match; COMT: caffeic acid O-methyltransferase; ACP: Acyl carrier protein; SAM: S-adenosyl-L-methionine; MFS: major facilitator superfamily; FAD: flavin adenine dinucleotide; FMN: flavin mononucleotide; CO: carbon monoxide.
}

\subsection{Butyrolactone I Addition Reveals Opposite Gene Expression Profiles for the Pgm Cluster Genes and DOPA-Type Melanin Biosynthesis Genes}

Recently, an uncommon melanin biosynthesis pathway of DOPA-type in A. terreus has been revealed to be composed of an NRPS-like biosynthase MelA (ATEG_03563) and a tyrosinase TyrP (ATEG_03564) [17,18]. In the sequenced transcriptome data, both of these genes were also expressed (Figure S4), with tyrP transcripts having lower pooled accumulation level in comparison to melA and the majority of genes encoding the tailoring enzymes of the $p g m$ cluster (Table 1). The observation that both of these gene clusters were expressed in the growth conditions used in this study led to a closer investigation of the previously obtained and accuracy improved in-depth microarray gene expression results. The microarray accuracy was improved on a probe sequence level, which was evaluated with the transcriptome sequence data as conducted in our related study [22]. Reliable gene 
expression data was available for six of the nine $p g m$ cluster genes and both of the two DOPA-type melanin biosynthesis encoding genes (Figure 3). The addition of butyrolactone I, regardless of the time point of addition, resulted in opposite gene expression patterns of the asp-melanin and pgm cluster genes. The DOPA-type asp-melanin biosynthesis genes were increasingly downregulated towards the late growth phase: at $216 \mathrm{~h}$ post inoculation, it was statistically significant, whereas the $p g m$ cluster genes were statistically significantly upregulated at the end of late growth phase. In particular, the $p g m D$, putative short-chain dehydrogenase/reductase, was significantly upregulated with a $\log _{2} \mathrm{FC}$ value above 2 when butyrolactone I was added at $24 \mathrm{~h}$ post inoculation. Some of these $p g m$ cluster genes were also statistically significantly downregulated prior to the end of late growth phase, i.e., before $216 \mathrm{~h}$ p.i. (Figure 3).

Taken together, the observed gene expression patterns indicate both direct and non-direct regulative roles for butyrolactone I concerning both of these two clusters, in these submerged growth conditions. The supplementation during the exponential growth phase led to both direct and non-direct regulation (Figure $3 \mathrm{~A}$ ), whereas the addition appears to have effect in a non-direct manner with an interval of $120 \mathrm{~h}$ when butyrolactone I was added during the middle growth phase (at $96 \mathrm{~h}$ p.i.), while the addition during the late phase (at $120 \mathrm{~h}$ p.i.) resulted in an interval of $96 \mathrm{~h}$ before the displayed positive effect at $216 \mathrm{~h}$ p.i. (Figure $3 \mathrm{~B}, \mathrm{C}$ ). The melanin producing genes display a similar intervalled expression pattern with one remarkable exception: the regulation conducted by butyrolactone I appears as continuously negative. Furthermore, the effect of the added butyrolactone I was most significant at the late growth phase regardless of the time point of addition unifying the presumably opposite gene expression regulation between both gene clusters.

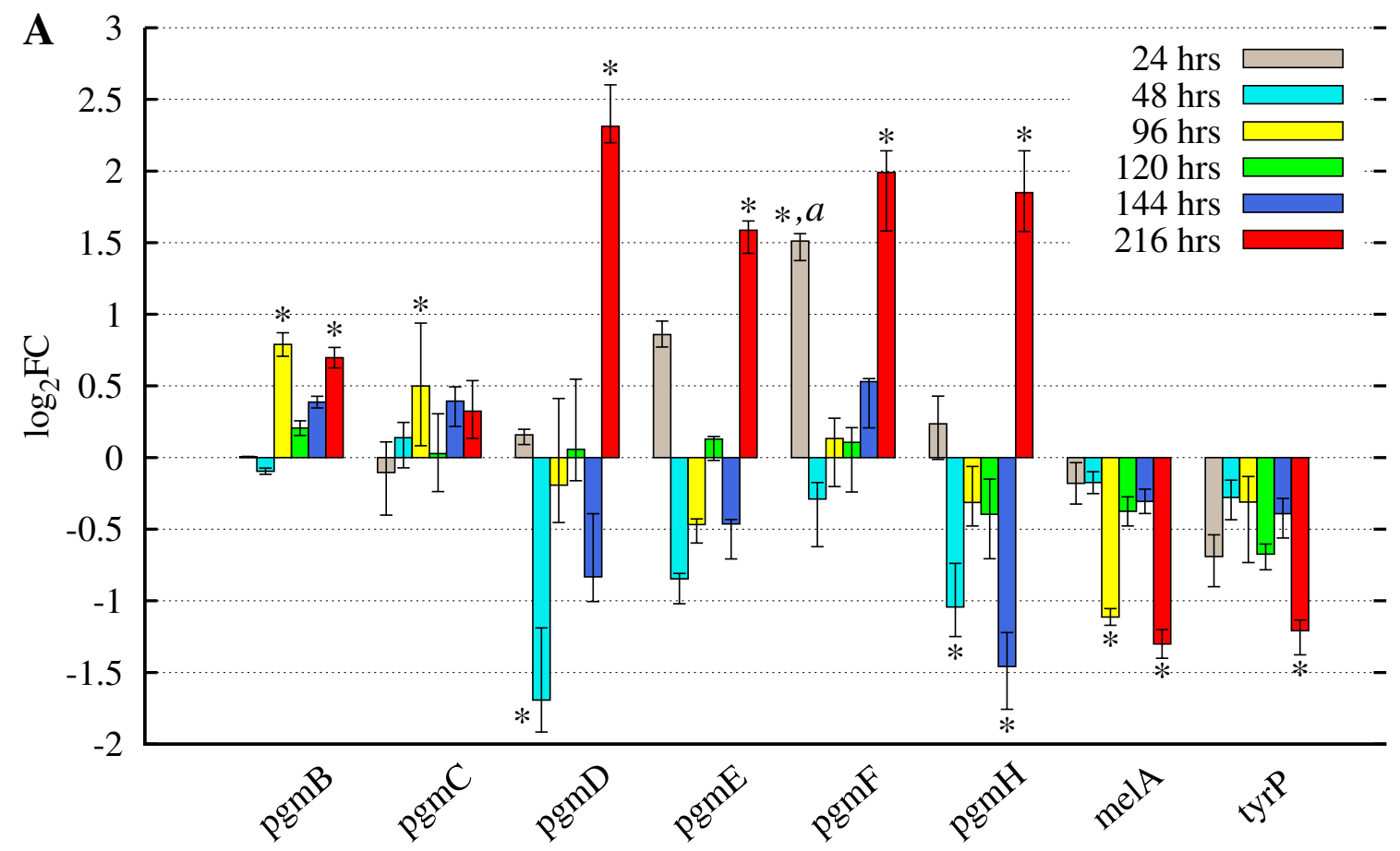

Figure 3. Cont. 


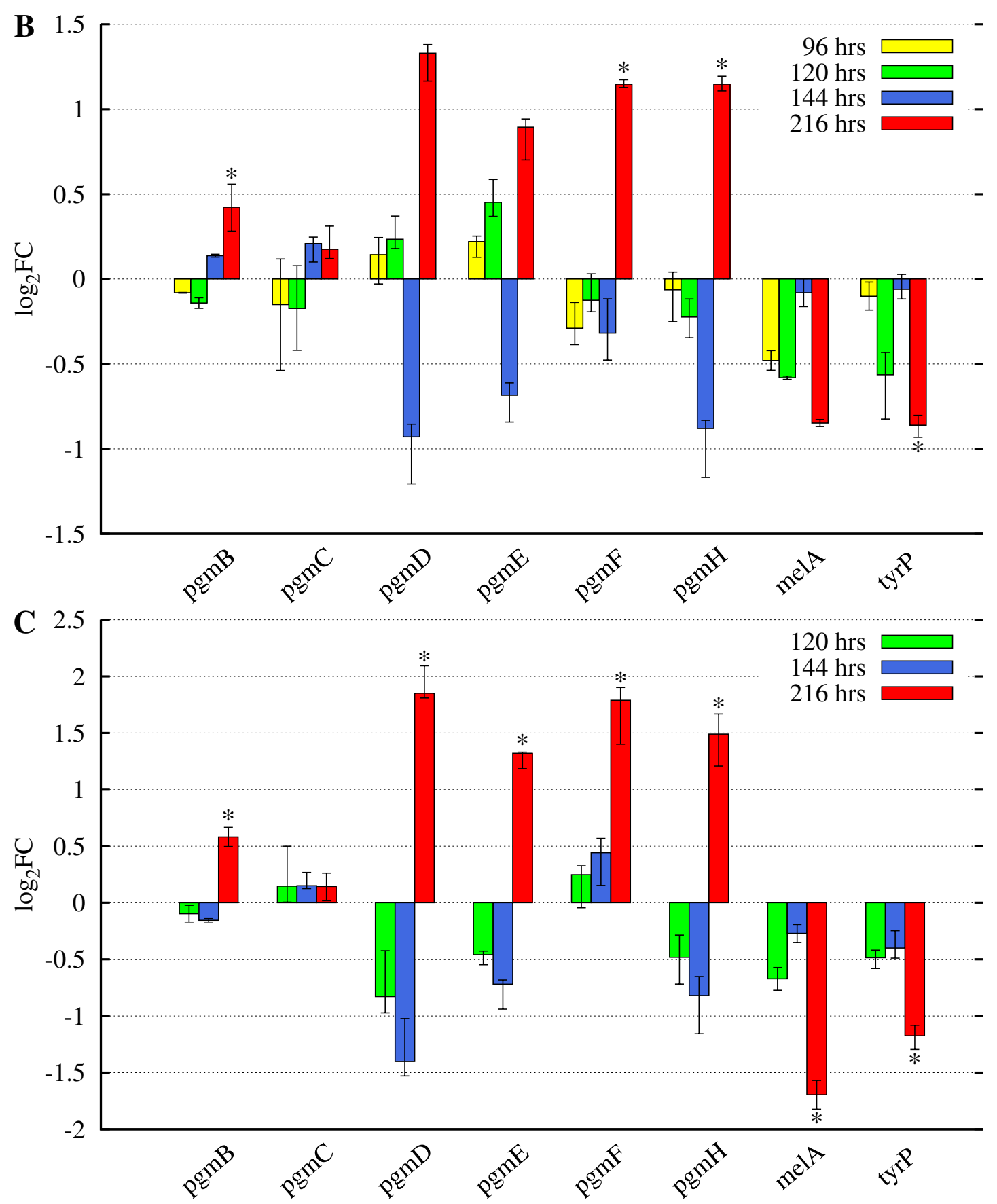

Figure 3. Gene expression analysis of the A. terreus pgm PKS cluster and asp-melanin genes under influence of butyrolactone I. Exogenous butyrolactone I was added to the submerged culture of A. terreus MUCL 38669 at (A) 24 h; (B) 96 h; and (C) 120 h p.i. to the final concentration of $100 \mathrm{nM}$. The lack of some cluster genes is due to the nucleotide level differences between strains MUCL 38669 and NIH2624 or the low transcript coverage obtained. The bars represent the median values of the $\log _{2} \mathrm{FC}$ of treated samples (butyrolactone I was added) versus control samples (no butyrolactone I added) and the error bars represent the maxima and minima of these $\log _{2} \mathrm{FC}$ values. ${ }^{*}$ At least one of the technical replicates indicates statistically significant up- or downregulation of the three biological replicates (adjusted $p$-value $\leq 0.05$ and $\left|\log _{2} \mathrm{FC}\right| \geq 0.5$ ). ${ }^{a}$ The apparently statistically significant upregulation at $24 \mathrm{~h}$ post inoculation may not be biologically significant due to the low culture density at the start of exponential growth phase. 


\section{Discussion}

\subsection{The Similarity of A. terreus Pgm Cluster with Pigment Biosynthesis Genes of Aspergilli and F. fujikuroi}

Fungal melanins are often complex polymers and divided into two different types (DHN and DOPA), depending on the characteristics of the biosynthesis pathways, being either produced intermediates or involved enzymes. Regarding Aspergillus species, the DHN-type of pigments are presumed to be more common, but DOPA-type of melanins have also been observed. A. fumigatus contains a DHN-type of conidial pigment biosynthesis cluster including an NR-PKS Alb1/PksP (with domains SAT-KS-AT-PT-ACP-ACP-TE/CLC) as well as Arp2, a hydroxynaphthalene reductase, common in this type of pigment cluster [41]. In A. nidulans, the homologous NR-PKS WA-with the same domains as $A$. fumigatus Alb1/PksP-has been shown to be involved in conidial pigmentation but is not genomically located in any specific cluster [11]. Both of these PKS enzymes accept malonyl-CoA as a precursor, whereas A. fumigatus cluster produces a pentaketide 1,3,6,8-tetrahydroxynaphthalene (T4HN; naphthalene-1,3,6,8-tetrol) to be polymerised into melanin, while A. nidulans produces YWA1 (heptaketide naphthopyrone) - another form of the initial precursor of the melanisation pathway of A. fumigatus [42,43]. A. flavus contains three separate PKS homologues of $A$. fumigatus Alb1/PksP, the most similar one ( $\mathrm{PksP}$ ) being in a similar gene cluster while the other two homologues are located in diverse gene clusters. One of them was shown to be the key polyketide synthase-domains SAT-KS-AT-PT-ACP-TE/CLC, having acetyl-CoA and malonyl-CoA as precursors-that produces putative anthraquinone intermediates as a presumed source for a sclerotia-specific pigment. The suggested pigment source was either a dehydrated form of anthraquinone asparasone A (1,3,6,8-tetrahydroxy-2-(1'-hydroxy-3'-oxobutyl)-anthraquinone) or a reduced form of anthraquinone [12]. There are also few DOPA-types of melanins reported to be biosynthesised, specifically in A. nidulans and A. flavus-with the intermediates and precursors remaining unknown $[13,14]$, whereas $A$. fumigatus has been observed to produce pyomelanin through a tyrosine degradation pathway, having benzoquinoneacetate as the precursor for polymerisation [44]. A. terreus has been reported to biosynthesise aspulvinone E-derived melanin by an NRPS-like enzyme (MelA) with 4-hydroxyphenylpyruvate as a precursor and subsequent hydroxylation of aspulvinone $\mathrm{E}$ ((5Z)-4-hydroxy-3-(4-hydroxyphenyl)-5-[(4-hydroxyphenyl)methylidene]furan-2-one) by tyrosinase (TyrP) prior to polymerisation $[17,18]$, classifying the Asp-melanin as DOPA-type.

Amongst Ascomycota, the NR-PKS enzymes have been divided into seven different groups, two of which contain enzymes involved in the biosynthesis of melanins and pigments [45]. When these groups are phylogenetically analysed together with the NR-PKS enzymes included in the phylogram presented in the study of Studt et al. [37] (Figure 2), an additional group within NR-PKS enzymes is formed containing several enzymes involved in the biosynthesis of pigments and melanins of Fusarium species, fusarubin included, and the asparasone A-derived pigment of $A$. flavus. Furthermore, the predicted PgmA of $A$. terreus appears to be part of the additional group (Figure 4 and Figure S5). The majority of the members of this additional group share the predicted domain structure-SAT-KS-AT-ACP-ACP with the thioester reductase $(\mathrm{R})$ domain on the C-terminus-resulting in a terminal aldehyde on the intermediate (heptaketide naphthaldehyde; 3-acetonyl-1,6,8-trihydroxy-2-naphthaldehyde (1)) of the fusarubin biosynthesis pathway as proposed by Awakawa et al. [38] and Studt et al. (Figure 4) [37]. Thus far, this reductive release mechanism appears to be rather uncommon amongst the non-reducing PKS enzymes of Aspergillus species—especially regarding the known pigment biosynthesis pathways. The $A$. terreus pgm cluster with the presumed core NR-PKS gene as described in this study contains also a putative short-chain dehydrogenase/reductase gene $(p g m D)$ with predicted classical SDR family and NADB_Rossmann superfamily protein domains (Table 2), displaying similarity with the domains of A. fumigatus Arp2 of the pigment cluster that contains the core PKS Alb1 [41]. The domain similarity of encoded PgmD with this common hydroxynaphthalene reductase as well as the same domain structure of the predicted PgmA in comparison to the F. fujikuroi Fsr1, the core NR-PKS enzyme of 
the fusarubin pigment cluster [37], sparks a hypothesis of this A. terreus pgm cluster to produce a DHN-type of pigment.

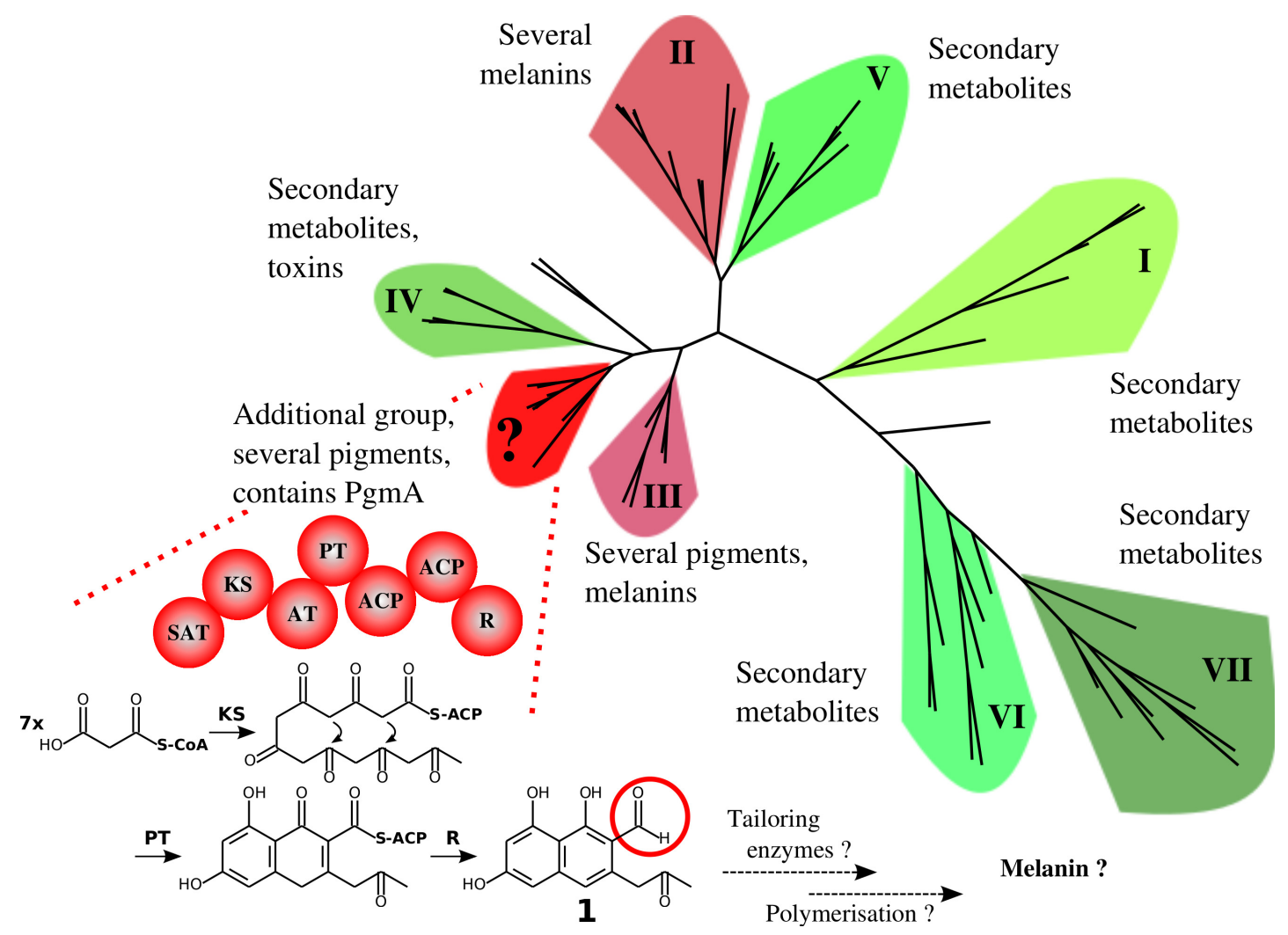

Figure 4. A phylogenetic scheme of various non-reducing polyketide synthases amongst Ascomycota phylum, dividing into eight groups based on the similarity of the ketosynthase domain sequences. The suggested hypothetical action mechanism for the majority of the NR-PKS enzymes amongst the additional group is as proposed by Awakawa et al., resulting in the intermediate 1; 3-acetonyl-1,6,8-trihydroxy-2-naphthaldehyde [38]. See a more detailed Figure S5 for the included NR-PKS proteins. The group classification of I-VII is based on the classes presented in the study of Ahuja et al., and the range of NR-PKS enzymes included in this analysis is based on the studies of Studt et al. and Ahuja et al. [37,45]. The extracted ketoacyl synthase domains of the polyketide synthases were compared and aligned to obtain the phylogram as described in our related study [22]. No outgroup was used. The protein sequences were obtained from the following databases: NCBI RefSeq, GenBank, DDBJ, EMBL and UniProtKB.

\subsection{Hypothesised A. terreus DHN-Type of Pigment and the Known DOPA-Type of Melanin May Be Biosynthesised under Different Growth Conditions}

In contrast to the observed DOPA-type melanin in A. terreus [17,18], Pal et al. [14] has reported melanin production to occur through the DHN pathway. This was demonstrated by adding the hydroxynaphthalene reductase inhibitors, tricyclazole and phthalide, which resulted in albino culture during static growth conditions, while the actual gene cluster still remained unknown. Melanin was also detected in the filtrate of submerged culture while no melanin was detected in the culture biomass. In addition, no production of DOPA-type of melanin was detected by using inhibitors kojic acid and tropolone [14]. In contradiction, the effects of deletion of a DOPA pathway gene $(m e l A)[17,18]$ and the addition of the DHN inhibitors [14] were both observed on static culture conditions to result in albino culture in both cases, which implies two separate melanin pigment biosynthesis pathways for A. terreus. This is due to the fact that no effect was observed when DOPA inhibitors were added in the same growth conditions as DHN inhibitors were added [14]. In particular, the observation that DOPA 
inhibitors had no effect during static growth conditions by Pal et al. [14], while the observations by Guo et al. and Geib et al. $[17,18]$ indicate the presence of DOPA pathway on static culture conditions, emphasises the importance of the growth conditions including production media used in melanin production. In this study, the used culture conditions being shaken and submerged, with exogenous addition and enhanced production of butyrolactone I (demonstrated in [20,21]), result in both pgm and asp-melanin clusters being expressed on a transcript level with opposing gene expression patterns (Figure 3, Figures S1, S2 and S4). These controversial gene expression profiles support the hypothesis of biogenesis of a DHN-type pigment by the $p g m$ cluster.

\subsection{The Suggested DHN-Like Pigment of A. terreus May Be Related to Conidiophores as Well}

In our related study [22], the gene expression patterns of the conidiation regulating genes, $a b a A$ and $w e t A$, are described to be negatively regulated prior to the end of last growth phase, while $a b a A$ is positively regulated at the last growth phase, $216 \mathrm{~h}$ p.i., in the same growth conditions as used in this study. The gene expression profiles of the presumed pigment cluster $p g m$ (Figure 3) are quite similar with especially the gene expression pattern of the phialide emergence inducing gene, $a b a \mathrm{~A}$, whereas the gene expression profiles of the DOPA-type Asp-melanin biosynthesis genes have contradictory forms. This indicates the predicted DHN-like pigment to be biosynthesised during the last steps of conidiation, phialide development followed by conidia maturation, while the Asp-melanin is presumably produced in a lesser amount. The gene expression profile and the convenient chronological order led us to further hypothesise regarding the morphological location of the suggested DHN-like melanin to be in the conidiophores in these submerged and shaken conditions under enhanced butyrolactone I biogenesis. This is in accordance with an observation of increasingly brown submerged culture towards the late phase, i.e., the ninth day of growth (unpublished observation). However, further studies are required to confirm this.

The question whether this $p g m$ gene cluster produces a DHN-like pigment, instead of some unrevealed secondary metabolite, may be explained by the gene expression profile of the secondary metabolism inducing LaeA, which was displayed in our related study [22]. The obtained laeA gene expression profile appears to be chronologically opposed to the revealed expression pattern of $p g m$ cluster (Figure 3). In our related study, laeA displayed significant upregulation by butyrolactone I prior to the late growth phase (at 48, 120 and $144 \mathrm{~h}$ p.i.), while it was unaffected at the late growth phase ( $216 \mathrm{~h}$ p.i.), indicating the increased secondary metabolism to occur prior to enhancing the gene expression of the $p g m$ cluster. The biogenesis of the secondary metabolite lovastatin has been observed to be increased by butyrolactone I during the middle growth phase in the same growth conditions as used in this study $[19,20]$, in agreement with the upregulated laeA gene expression profile [22]. Considering these observed occurrence patterns in chronological order, the activity of the pgm cluster appears to be enhanced after the increase in secondary metabolism in these submerged culture conditions under the influence of increased butyrolactone I biogenesis.

\section{Conclusions}

In conclusion, we provide a hypothesis suggesting the new, non-canonical NR-PKS PgmA amongst Aspergilli, being an ortholog of F. fujikuroi fusarubin core synthase, Fsr1, to be a key pigment biosynthase with the reductase domain on the C-terminus. In addition, we suggest the cluster in the vicinity of this core gene, $\operatorname{pgmA}$, to contain a hydroxynaphthalene reductase-like short-chain dehydrogenase/reductase PgmD, along with five co-expressed tailoring enzymes. Integrated with our related study of $A$. terreus conidiation during submerged culture, the results of this study indicate increased biogenesis of a conidia related DHN-type of pigment under submerged growth conditions with increased butyrolactone I production. To confirm the obtained results and this hypothesised conidial pigment production, further comprehensive studies including quantitative RNA-sequencing and molecular as well as morphological analyses are necessary. 
Supplementary Materials: The following are available online at http://www.mdpi.com/2076-2607/5/2/22/s1. Figure S1: Sashimi plots of $p g m B, p g m C, p g m R$ and $p g m D$ genes of the $p g m$ PKS cluster, Figure S2: Sashimi plots of $p g m E, p g m F, p g m G$ and $p g m H$ genes of the pgm PKS cluster, Figure S3: Sashimi plots of ATEG_06202 and ATEG_06212, Figure S4: Sashimi plots of the NRPS-like melanin biosynthase melA and tyrosinase tyrP, Figure S5: Detailed version of the phylogram in the Figure 4.

Acknowledgments: This work was supported by a European Commission grant, STREP-FP6-NMP4-CT2006-032811, under the Sixth Framework Project QUORUM. E.K.P. acknowledges the National Doctoral Programme in Informational and Structural Biology and Stiftelsen för Liv och Hälsa for funding and the Finnish IT Center for Science for providing the IT infrastructure for the data analysis.

Author Contributions: T.K., J.T.S., A.B. and J.M. conceived and designed the experiments and provided supervision; E.K.P. and S.R. performed the experiments; E.K.P. analyzed the data; A.B., J.T.S. and T.K. contributed reagents and materials; and E.K.P. wrote the paper.

Conflicts of Interest: The authors declare no conflict of interest.

\section{Abbreviations}

The following abbreviations are used in this manuscript:

$\begin{array}{ll}\text { DHN } & \text { 1,8-dihydroxynaphthalene } \\ \text { DOPA } & \text { 3,4-dihydroxyphenylalanine } \\ \text { FPKM } & \text { fragments per kilobase of exon per million reads mapped } \\ \text { ORF } & \text { open reading frame } \\ \text { p.i. } & \text { post inoculation } \\ \text { FC } & \text { fold change } \\ \text { PKS } & \text { polyketide synthase } \\ \text { SAT } & \text { starter unit: ACP transacylase } \\ \text { KS } & \text { Beta-ketoacyl synthase } \\ \text { AT } & \text { Acyl transferase } \\ \text { PT } & \text { Polyketide product template } \\ \text { ACP } & \text { Acyl carrier } \\ \text { R } & \text { Thioester reductase }\end{array}$

\section{References}

1. Volling, K.; Thywissen, A.; Brakhage, A.A.; Saluz, H.P. Phagocytosis of melanized Aspergillus conidia by macrophages exerts cytoprotective effects by sustained PI3K/Akt signalling. Cell Microbiol. 2011, 13, 1130-1148.

2. Rosa, L.H.; de Lourdes Almeida Vieira, M.; Santiago, I.F.; Rosa, C.A. Endophytic fungi community associated with the dicotyledonous plant Colobanthus quitensis (Kunth) Bartl. (Caryophyllaceae) in Antarctica. FEMS Microbiol. Ecol. 2010, 73, 178.

3. Zalar, P.; Novak, M.; de Hoog, G.; Gunde-Cimerman, N. Dishwashers-A man-made ecological niche accommodating human opportunistic fungal pathogens. Fungal Biol. 2011, 115, 997-1007.

4. Lass-Flörl, C.; Kofler, G.; Kropshofer, G.; Hermans, J.; Kreczy, A.; Dierich, M.P.; Niederwieser, D. In-vitro testing of susceptibility to amphotericin B is a reliable predictor of clinical outcome in invasive aspergillosis. J. Antimicrob. Chemother. 1998, 42, 497.

5. Lass-Flörl, C.; Griff, K.; Mayr, A.; Petzer, A.; Gastl, G.; Bonatti, H.; Freund, M.; Kropshofer, G.; Dierich, M.P.; Nachbaur, D. Epidemiology and outcome of infections due to Aspergillus terreus: 10-Year single centre experience. Br. J. Haematol. 2005, 131, 201-207.

6. Eisenman, H.C.; Casadevall, A. Synthesis and assembly of fungal melanin. Appl. Microbiol. Biotechnol. 2011, 93, 931-940.

7. Wheeler, M.; Klich, M. The effects of tricyclazole, pyroquilon, phthalide, and related fungicides on the production of conidial wall pigments by Penicillium and Aspergillus species. Pestic. Biochem. Physiol. 1995, 52, 125-136.

8. Cabanes, J.; Chazarra, S.; Garcia-Carmona, F. Kojic acid, a cosmetic skin whitening agent, is a slow-binding inhibitor of catecholase activity of tyrosinase. J. Pharm. Pharmacol. 1994, 46, 982-985.

9. Espín, J.C.; Wichers, H.J. Slow-binding inhibition of mushroom (Agaricus bisporus) tyrosinase isoforms by tropolone. J. Agric. Food Chem. 1999, 47, 2638-2644.

10. Chang, T.S. An updated review of tyrosinase inhibitors. Int. J. Mol. Sci. 2009, 10, 2440-2475. 
11. Mayorga, M.E.; Timberlake, W.E. Isolation and molecular characterization of the Aspergillus nidulans wA gene. Genetics 1990, 126, 73-79.

12. Cary, J.W.; Harris-Coward, P.Y.; Ehrlich, K.C.; Mavungu, J.D.D.; Malysheva, S.V.; Saeger, S.D.; Dowd, P.F.; Shantappa, S.; Martens, S.L.; Calvo, A.M. Functional characterization of a veA-dependent polyketide synthase gene in Aspergillus flavus necessary for the synthesis of asparasone, a sclerotium-specific pigment. Fungal Genet. Biol. 2014, 64, 25-35.

13. Gonçalves, R.C.R.; Lisboa, H.C.F.; Pombeiro-Sponchiado, S.R. Characterization of melanin pigment produced by Aspergillus nidulans. World J. Microbiol. Biotechnol. 2011, 28, 1467-1474.

14. Pal, A.K.; Gajjar, D.U.; Vasavada, A.R. DOPA and DHN pathway orchestrate melanin synthesis in Aspergillus species. Med. Mycol. 2014, 52, 10-18.

15. Zaehle, C.; Gressler, M.; Shelest, E.; Geib, E.; Hertweck, C.; Brock, M. Terrein biosynthesis in Aspergillus terreus and its impact on phytotoxicity. Chem. Biol. 2014, 21, 719-731.

16. Gressler, M.; Meyer, F.; Heine, D.; Hortschansky, P.; Hertweck, C.; Brock, M. Phytotoxin production in Aspergillus terreus is regulated by independent environmental signals. eLife 2015, 4, e07861.

17. Guo, C.J.; Knox, B.P.; Sanchez, J.F.; Chiang, Y.M.; Bruno, K.S.; Wang, C.C.C. Application of an efficient gene targeting system linking secondary metabolites to their biosynthetic genes in Aspergillus terreus. Org. Lett. 2013, 15, 3562-3565.

18. Geib, E.; Gressler, M.; Viediernikova, I.; Hillmann, F.; Jacobsen, I.D.; Nietzsche, S.; Hertweck, C.; Brock, M. A non-canonical melanin biosynthesis pathway protects Aspergillus terreus conidia from environmental stress. Cell Chem. Biol. 2016, 23, 587-597.

19. Schimmel, T.G.; Coffman, A.D.; Parsons, S.J. Effect of butyrolactone I on the producing fungus, Aspergillus terreus. Appl. Environ. Microbiol. 1998, 64, 3707-3712.

20. Raina, S.; de Vizio, D.; Palonen, E.K.; Odell, M.; Brandt, A.M.; Soini, J.T.; Keshavarz, T. Is quorum sensing involved in lovastatin production in the filamentous fungus Aspergillus terreus? Process. Biochem. 2012, 47, 843-852.

21. Palonen, E.K.; Neffling, M.R.; Raina, S.; Brandt, A.; Keshavarz, T.; Meriluoto, J.; Soini, J. Butyrolactone I quantification from lovastatin producing Aspergillus terreus using tandem mass spectrometry-Evidence of signalling functions. Microorganisms 2014, 2, 111-127.

22. Palonen, E.K.; Raina, S.; Brandt, A.; Meriluoto, J.; Keshavarz, T.; Soini, J.T. Transcriptomic complexity of Aspergillus terreus velvet gene family under the influence of butyrolactone I. Microorganisms 2017, 5, 12.

23. Birren, B.; Lander, E.; Galagan, J.; Nusbaum, C.; Devon, K.; Henn, M.; Ma, L.J.; Jaffe, D.; Butler, J.; Alvarez, P.; et al. Annotation of the Aspergillus terreus NIH2624 Genome. Available online: http: //www.broadinstitute.org/ftp/pub/annotation/fungi/aspergillus/genomes/ (accessed on 9 March 2007).

24. Nucleotide-Nucleotide BLAST Version 2.2.29+. Available online: ftp://ftp.ncbi.nlm.nih.gov/blast/ executables/blast+/2.2.29/ (accessed on 10 October 2014).

25. Camacho, C.; Coulouris, G.; Avagyan, V.; Ma, N.; Papadopoulos, J.; Bealer, K.; Madden, T.L. BLAST+: Architecture and applications. BMC Bioinform. 2009, 10, 1-9.

26. Marioni, J.C.; Mason, C.E.; Mane, S.M.; Stephens, M.; Gilad, Y. RNA-seq: An assessment of technical reproducibility and comparison with gene expression arrays. Genome Res. 2008, 18, 1509-1517.

27. Parkhomchuk, D.; Borodina, T.; Amstislavskiy, V.; Banaru, M.; Hallen, L.; Krobitsch, S.; Lehrach, H.; Soldatov, A. Transcriptome analysis by strand-specific sequencing of complementary DNA. Nucleic Acids Res. 2009, 37, e123.

28. Levin, J.Z.; Yassour, M.; Adiconis, X.; Nusbaum, C.; Thompson, D.A.; Friedman, N.; Gnirke, A.; Regev, A. Comprehensive comparative analysis of strand-specific RNA sequencing methods. Nat. Methods 2010, 7, 709-715.

29. Integrative Genomics Viewer Software. Version IGV_2.3.26. Available online: http:/ / software.broadinstitute. org/software/igv/ (accessed on 31 January 2014).

30. Robinson, J.T.; Thorvaldsdóttir, H.; Winckler, W.; Guttman, M.; Lander, E.S.; Getz, G.; Mesirov, J.P. Integrative genomics viewer. Nat. Biotechnol. 2011, 29, 24-26.

31. Thorvaldsdóttir, H.; Robinson, J.T.; Mesirov, J.P. Integrative genomics viewer (IGV): High-performance genomics data visualization and exploration. Brief Bioinform. 2013, 14, 178-192. 
32. Trapnell, C.; Williams, B.A.; Pertea, G.; Mortazavi, A.; Kwan, G.; van Baren, M.J.; Salzberg, S.L.; Wold, B.J.; Pachter, L. Transcript assembly and quantification by RNA-seq reveals unannotated transcripts and isoform switching during cell differentiation. Nat. Biotechnol. 2010, 28, 511-515.

33. The GENSCAN Web Server at MIT. Available online: http://genes.mit.edu/GENSCAN.html (accessed on 1 January 2015).

34. ExPASy - Translate Tool. Available online: http://web.expasy.org/translate/ (accessed on 1 January 2015).

35. InterProScan Sequence Search. Available online: http://www.ebi.ac.uk/interpro/search/sequence-search/ (accessed on 1 January 2015).

36. Basic Local Alignment Tool, NCBI BLAST Sequence Database. Available online: http://blast.ncbi.nlm.nih. gov/ (accessed on 1 January 2015).

37. Studt, L.; Wiemann, P.; Kleigrewe, K.; Humpf, H.U.; Tudzynski, B. Biosynthesis of fusarubins accounts for pigmentation of Fusarium fujikuroi perithecia. Appl. Environ. Microbiol. 2012, 78, 4468-4480.

38. Awakawa, T.; Kaji, T.; Wakimoto, T.; Abe, I. A heptaketide naphthaldehyde produced by a polyketide synthase from Nectria haematococca. Bioorg. Med. Chem. Lett. 2012, 22, 4338-4340.

39. Mulder, N.J.; Apweiler, R.; Attwood, T.K.; Bairoch, A.; Bateman, A.; Binns, D.; Bork, P.; Buillard, V.; Cerutti, L.; Copley, R.; et al. New developments in the InterPro database. Nucleic Acids Res. 2007, 35, D224-D228.

40. Jones, P.; Binns, D.; Chang, H.Y.; Fraser, M.; Li, W.; McAnulla, C.; McWilliam, H.; Maslen, J.; Mitchell, A.; Nuka, G.; et al. InterProScan 5: Genome-scale protein function classification. Bioinformatics 2014, 30, 1236-1240.

41. Tsai, H.F.; Wheeler, M.H.; Chang, Y.C.; Kwon-Chung, K.J. A developmentally regulated gene cluster involved in conidial pigment biosynthesis in Aspergillus fumigatus. J. Bacteriol. 1999, 181, 6469-6477.

42. Tsai, H.F.; Fujii, I.; Watanabe, A.; Wheeler, M.H.; Chang, Y.C.; Yasuoka, Y.; Ebizuka, Y.; Kwon-Chung, K.J. Pentaketide melanin biosynthesis in Aspergillus fumigatus requires chain-length shortening of a heptaketide precursor. J. Biol. Chem. 2001, 276, 29292-29298.

43. Fujii, I.; Yasuoka, Y.; Tsai, H.F.; Chang, Y.C.; Kwon-Chung, K.J.; Ebizuka, Y. Hydrolytic polyketide shortening by Ayg1p, a novel enzyme involved in fungal melanin biosynthesis. J. Biol. Chem. 2004, 279, 44613-44620.

44. Keller, S.; Macheleidt, J.; Scherlach, K.; Schmaler-Ripcke, J.; Jacobsen, I.D.; Heinekamp, T.; Brakhage, A.A. Pyomelanin formation in Aspergillus fumigatus requires $\mathrm{HmgX}$ and the transcriptional activator HmgR but is dispensable for virulence. PLoS ONE 2011, 6, 1-11.

45. Ahuja, M.; Chiang, Y.M.; Chang, S.L.; Praseuth, M.B.; Entwistle, R.; Sanchez, J.F.; Lo, H.C.; Yeh, H.H.; Oakley, B.R.; Wang, C.C.C. Illuminating the diversity of aromatic polyketide synthases in Aspergillus nidulans. J. Am. Chem. Soc. 2012, 134, 8212-8221.

(C) 2017 by the authors. Licensee MDPI, Basel, Switzerland. This article is an open access article distributed under the terms and conditions of the Creative Commons Attribution (CC BY) license (http:/ / creativecommons.org/licenses/by/4.0/). 